\title{
Adrenal haemorrhage and infarction in the setting of vaccine-induced immune thrombocytopenia and thrombosis after SARS-CoV-2 (Oxford-AstraZeneca) vaccination
}

\author{
Anneke Graf(iD), Eleni Armeni(D1, Louise Dickinson2, Matthew Stubbs33, Brian Craven³, \\ Umasuthan Srirangalingam ${ }^{1}$ and Teng-Teng Chung ${ }^{1}$
}

1Department of Endocrinology, 2Department of Radiology, and ${ }^{3}$ Department of Haematology, University College London Hospitals NHS Foundation Trust, London, UK
Correspondence should be addressed to T-T Chung

Email

teng-teng.chung@nhs.net

\section{Summary}

Rare cases of vaccine-induced Immune thrombocytopenia and thrombosis (VITT) are being identified after vaccination with the SARS-CoV-2 Oxford-AstraZeneca vaccination. We report on two such patients with associated adrenal involvement, which is now being recognised. Both patients presented with abdominal pain, back pain and vomiting. Case 1 was a 46-year-old male who had received the first dose of the Oxford-AstraZeneca vaccination 8 days earlier. Imaging demonstrated a number of serious thrombotic complications including evolving bilateral adrenal haemorrhage (right adrenal haemorrhage identified at presentation, with the left-sided changes only evident on day 4 of the admission). Case 2 was a 38-year-old female who had received the first dose of Oxford-AstraZeneca vaccination 11 days prior. Imaging demonstrated left renal vein thrombosis and left adrenal infarction. VITT was diagnosed in both cases given these changes and other consistent haematological findings. Both patients were treated empirically for adrenal insufficiency, a diagnosis subsequently confirmed in case 1 . We report these two cases of VITT presenting with adrenal complications (haemorrhage and infarction) after Oxford-AstraZeneca vaccination to highlight the association and the need for prompt management of co-existing adrenal insufficiency, especially given the potential for evolving adrenal involvement.

\section{Learning points:}

- Adrenal complications (thrombosis/infarction/haemorrhage) may develop as a part of vaccine-induced immune thrombocytopenia (VITT) after SARS-CoV-2 Oxford-AstraZeneca vaccination.

- Evolving adrenal involvement is possible and ongoing assessment is required to identify this promptly.

- Cortisol levels may be difficult to interpret when assessing for adrenal insufficiency, given high doses of corticosteroids may be used to manage VITT.

- Clinicians should have a low threshold for starting empirical replacement with corticosteroids until reliable assessment of adrenal function can be performed.

\section{Background}

While vaccination against severe acute respiratory syndrome coronavirus 2 (SARS-CoV-2) with the ChAdOx1 nCoV-19 (Oxford-AstraZeneca) vaccination is safe and effective (1), rare cases of vaccine-induced immune thrombocytopenia and thrombosis (VITT) are being identified (2). We report two cases of VITT after 
Oxford-AstraZeneca vaccination presenting with adrenal haemorrhage (case 1) and adrenal infarction (case 2), highlighting the association and the need for prompt management of co-existing adrenal insufficiency.

\section{Case presentation and investigations}

Case 1

A 46-year-old Caucasian male presented to the emergency department with a 24-h history of abdominal and back pain. He was previously fit and well, taking no regular medications. He was a current smoker of 27 pack years and drank up to 16 units of alcohol per day. He had received the first dose of SARS-CoV-2 vaccine (Oxford-AstraZeneca) 8 days earlier.

The pain was dull and aching in nature, experienced primarily in the epigastric and umbilical regions of the abdomen and also bilaterally in the lower back. There was associated nausea and vomiting.

A computerised tomography (CT) scan of the abdomen and pelvis demonstrated an enlarged, hyperdense right adrenal gland with surrounding fat stranding, consistent with acute haemorrhage (see Fig. 1A). There was no known history of pre-existing adrenal pathology.

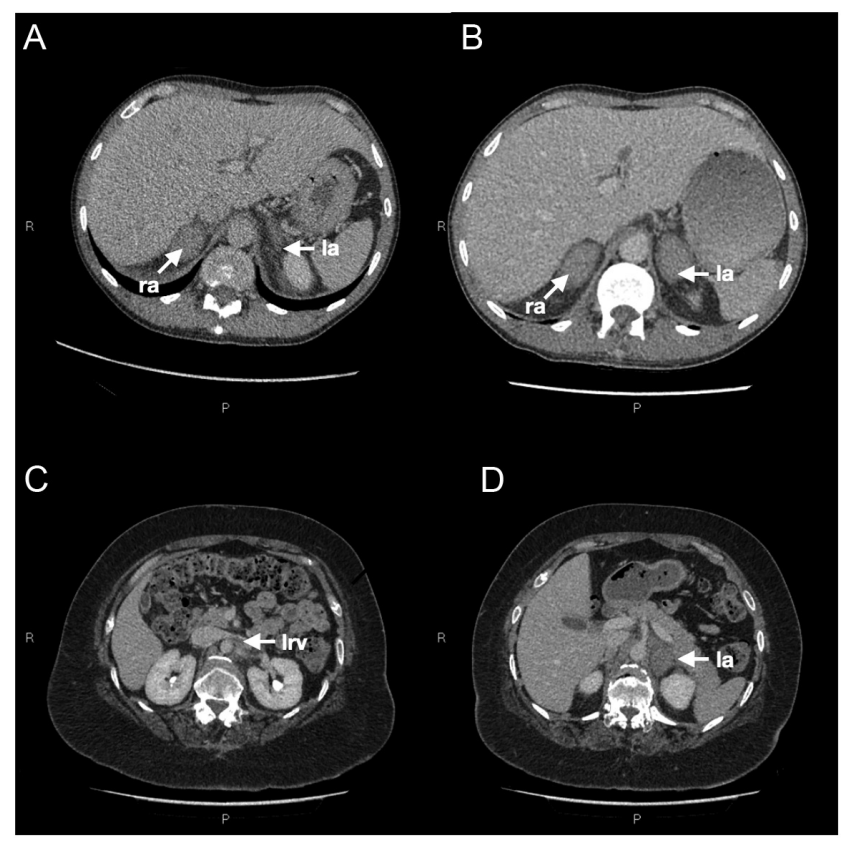

\section{Figure 1}

Computerised tomography (CT) images for case 1 and 2. (A) ^ Case 1: right adrenal haemorrhage, left adrenal normal appearance (day 1). (B) *Case 1: bilateral adrenal haemorrhage (day 4). (C)^Case 2: left renal vein thrombosis (day 1). (D) ^ Case 2: left adrenal infarction (day 1). ra, right adrenal gland; la, left adrenal gland, Irv, left renal vein. ${ }^{\wedge} \mathrm{CT}$ abdomen/ pelvis with contrast, ${ }^{\star} \mathrm{CT}$ angiogram pulmonary.
Regarding clinical evidence of adrenal insufficiency, there was no haemodynamic compromise and biochemistry demonstrated normal sodium, potassium, acid-base status and glucose levels. A baseline random cortisol (taken at 13:40 h) was $87 \mathrm{nmol} / \mathrm{L}$ and this confirmed adrenal insufficiency in an acutely unwell subject.

A diagnosis of VITT (3) was made over the following days given the development of numerous serious thrombotic complications, worsening thrombocytopenia and other haematological findings consistent with this diagnosis (see Table 1).

A CT pulmonary angiogram performed on day 4 of the admission, in the setting of investigation of chest pain, revealed a new left-sided adrenal haemorrhage. This finding was not present on initial imaging when the right adrenal haemorrhage had been first identified (see Fig. 1B).

\section{Case 2}

A 38-year-old Asian female presented to the emergency department with a 48-h history of abdominal and back pain. Her past medical history included gastro-oesophageal reflux disease, osteoarthritis, obesity (BMI $38.8 \mathrm{~kg} / \mathrm{m}^{2}$ ) and spondyloepiphyseal dysplasia with previous joint replacements and spinal surgeries. She had received the first dose of SARS-CoV-2 vaccine (Oxford-AstraZeneca) 11 days earlier.

This was her fourth presentation to hospital, with assessment at three other emergency departments failing to identify the cause of her symptoms. The abdominal pain was initially generalised but then localised to the upper abdomen and radiated to the back. She also experienced a severe headache and vomiting.

A CT abdomen and pelvis demonstrated a filling defect in the distal portion of the left renal vein consistent with thrombosis and an enlarged left adrenal gland, with loss of shape and decreased attenuation, in keeping with evolving infarction and post-infarction necrosis (see Fig. 1C and D). The right adrenal gland appeared normal. Again, there was no prior history of adrenal pathology. There was no clinical or biochemical evidence of adrenal insufficiency.

A diagnosis of VITT was made given the radiologically evident thrombosis in the left renal vein and a number of haematological findings (see Table 1).

A repeat CT scan on day 4 of the admission was again consistent with left adrenal infarction without evidence of haemorrhagic transformation. Despite the patient experiencing a headache, repeated cerebral imaging did not demonstrate any abnormalities, including evidence of venous sinus thrombosis. 
Table 1 Key details of cases 1 and 2.

Demographics
Presentation complaint
Time of presentation in relation to Oxford-
AstraZeneca vaccine
Radiological findings*

Biochemical findings of adrenal insufficiency (Normal peak cortisol >415 $\mathrm{nmol} / \mathrm{L}$ ); normal range ACTH: 7.2-63.3 ng/L)

Haematological findings consistent with VITT*\# Thrombocytopenia (Normal range: $150-400 \times 10 \% / L)$

Raised D-dimer (Normal range 0-550 $\mu \mathrm{g} / \mathrm{L} \mathrm{FEU)}$

PF4-induced antibodies (Positive if $>0.23$ optical density $(O D)$ )

Other complications of VITT*

\section{Case 1}

46-year-old male

$24 \mathrm{~h}$ of abdominal pain radiating to the

back, with nausea and vomiting

8 days post vaccination (dose 1 )

Bilateral adrenal haemorrhage

- Right: identified day 1

- Left: identified day 4

Short synacthen test:

(36 days after presentation)

- Basal cortisol: $16 \mathrm{nmol} / \mathrm{L}$

- Basal ACTH: 169.9 ng/L

- 30 min cortisol: $17 \mathrm{nmol} / \mathrm{L}$

- 60 min cortisol: $17 \mathrm{nmol} / \mathrm{L}$

Platelets day $0: 111 \times 10^{9 / L}$

Platelets day 4 (nadir): $11 \times 109 / \mathrm{L}$

Day 8 (peak): >80 $000 \mu \mathrm{g} / \mathrm{L}$ FEU

Day 7 (peak): 2.161 OD

- Day 2: acute inferior myocardial infarction

- Day 6: dural venous sinus thrombosis, right occipital lobe infarction

- Day 7: pulmonary embolism, portal vein thrombosis, hepatic vein thrombosis and bilateral renal cortical infarcts

\section{Case 2}

38-year-old female

$48 \mathrm{~h}$ of abdominal pain radiating

to the back, with headache and vomiting

11 days post vaccination (dose 1 )

Unilateral adrenal infarction (left)

Short synacthen test:

(98 days after presentation) ${ }^{\wedge}$

- Basal cortisol: $65 \mathrm{nmol} / \mathrm{L}$

- Basal ACTH: 12.6 ng/L

- 30 min cortisol: $239 \mathrm{nmol} / \mathrm{L}$

- 60 min cortisol: $295 \mathrm{nmol} / \mathrm{L}$

Platelets day 0: $102 \times 109 / \mathrm{L}$ Platelets day 1 (nadir): $72 \times 109 / \mathrm{L}$ Day 0 (peak): $4160 \mu \mathrm{g} / \mathrm{L} \mathrm{FEU}$ Day 0 (peak): 2.955 OD

- Persistently high levels of antibodies to PF4

${ }^{*}$ Days counted from day of admission (day 0); \#fibrinogen levels were not reduced (both patients); ${ }^{\wedge}$ performed after 6 weeks of high-dose corticosteroids required for another indication.

\section{Treatment}

Both patients required intensive care support and specialist haematology input. The management of VITT included pulse doses of i.v. methylprednisolone. For case 1, this was given day $6(1000 \mathrm{mg})$ and day $7(500 \mathrm{mg})$ of the admission. For case 2, $500 \mathrm{mg}$ was administered in the emergency department.

For case 1, i.v. hydrocortisone was administered empirically when adrenal involvement was first identified and then maintenance doses were continued. At the time of discharge to home on day 23 of the admission, steroid replacement had been weaned to a standard replacement dose of oral hydrocortisone (20 mg split throughout the day) and fludrocortisone had been commenced (100 $\mu \mathrm{g}$ per day) given the bilateral adrenal pathology.

For case 2, despite unilateral adrenal pathology, adrenal insufficiency could not be excluded. A morning $(6: 30 \mathrm{~h})$ serum cortisol level, taken when the patient was unwell in intensive care, was only $187 \mathrm{nmol} / \mathrm{L}$. The interpretation of this result was also difficult given methylprednisolone administration 3 days earlier. The patient was therefore discharged after an 8-day admission on empirical treatment with oral hydrocortisone $(20 \mathrm{mg}$ split throughout the day)

\section{Outcome and follow-up}

Further outpatient assessment of adrenal function was planned for both patients. For case 1, a short synacthen test, performed 12 days after discharge from hospital, confirmed primary adrenal insufficiency with a low basal (pre synacthen) cortisol of $16 \mathrm{nmol} / \mathrm{L}$; an elevated basal ACTH of $169 \mathrm{ng} / \mathrm{L}$ (normal range 7.2-63.3 ng/L), in the context of bilateral adrenal haemorrhage, and a peak cortisol of $17 \mathrm{nmol} / \mathrm{L}$ at both 30 and $60 \mathrm{~min}$ (normal level for peak cortisol $>415 \mathrm{nmol} / \mathrm{L})$. Aldosterone levels taken at the time of the basal sample were also low at $<60 \mathrm{pmol} / \mathrm{L}$ (normal range $90-700 \mathrm{pmol} / \mathrm{L}$ ), with a renin level of $3.1 \mathrm{nmol} / \mathrm{L} / \mathrm{h}$ (normal range $0.5-3.5 \mathrm{nmol} / \mathrm{L} / \mathrm{h}$ ). Hydrocortisone and fludrocortisone were continued. 
For case 2, a short synacthen test was delayed as the patient was subsequently commenced on high dose corticosteroids. There was thought to still be ongoing disease activity, given persistent symptoms (left flank pain) and high levels of antibodies to platelet factor 4 (PF4), the antibodies thought to mediate VITT (2). Prednisolone was slowly weaned over 6 weeks ( $60 \mathrm{mg}$ down to $5 \mathrm{mg}$ per day). A short synacthen test was then performed demonstrating an inadequate response, with a basal cortisol of $65 \mathrm{nmol} / \mathrm{L}$, a cortisol at $30 \mathrm{~min}$ of $239 \mathrm{nmol} / \mathrm{L}$ and a peak cortisol at $60 \mathrm{~min}$ of $295 \mathrm{nmol} / \mathrm{L}$. The basal ACTH was not elevated at $12.6 \mathrm{ng} / \mathrm{L}$ (normal range $7.2-63.3 \mathrm{ng} / \mathrm{L}$ ), consistent with unilateral adrenal pathology and exogenous glucocorticoids use. Aldosterone and renin levels were not assessed in the acute setting. The patient has commenced replacement oral hydrocortisone and another short synacthen test is planned to reassess adrenal function a few months after stopping prednisolone.

Repeat imaging was performed in case 2. A CT scan 6 weeks after discharge demonstrated reduction in the size of the left adrenal gland, which became more well defined with central hypodensity and peripheral enhancement. There was resolution of the previous peri-adrenal inflammatory change and the previously identified thrombosis of the left renal vein had resolved. Further reduction in size of the left adrenal lesion was again demonstrated on a MRI scan 7 weeks later.

\section{Discussion}

Guidelines from an expert haematology panel (3) describe VITT after Oxford-AstraZeneca vaccination occurring most commonly between 5 and 30 days and characterised by thrombocytopenia, raised D-dimer levels and thrombosis, which is often rapidly progressive. In particular, a high preponderance of cerebral vein thrombosis has been observed; however, complications affecting the adrenal gland, including haemorrhage and infarction, are now being reported.

The vaccine-induced prothrombotic state appears pathophysiologically similar to heparin-induced thrombocytopenia (HIT) $(4,5)$, which has previously been linked with adrenal haemorrhage (6). The rich arterial supply and comparatively poor venous outflow of the adrenal gland, usually with one central vein, have been highlighted as factors potentially increasing the risk of developing thrombotic infarction with haemorrhagic transformation within the gland $(6,7)$.

Seven cases of adrenal haemorrhage, one case of adrenal thrombosis and four cases of haemorrhagic adrenal infarction were reported after Oxford-AstraZeneca vaccination in the United Kingdom between January 4 and 4 August, 2021 (8). A recently published prospective cohort study of 220 patients with definite or probable VITT hospitalized in the United Kingdom between March 22 and June 6, 2021, reported adrenal complications of VITT (thrombosis or haemorrhage) in 3\% of cases (4). In this cohort, VITT was associated only with the first OxfordAstraZeneca vaccination and primarily seen in patients younger than 60 years of age. Multiple-site thromboses and arterial events were common.

Recently published case reports have highlighted four cases of bilateral adrenal haemorrhage in the setting of VITT after Oxford-AstraZeneca vaccination (9, 10, 11, 12). Similar to our experience, in two of these cases, symptoms related to the adrenal pathology were the presenting feature. In contrast, clinical features of adrenal insufficiency were detected 10 days after hospitalisation for VITT in the case described by Verona et al. (12), highlighting the importance of having an ongoing high index of suspicion for the development of adrenal pathology. Multiple complications of VITT were present in these cases, in keeping with the presentation of case 1 in our series. Unlike our experience, there was a high death rate observed in these case reports with two of the four patients dying of complications related to VITT. There appears to be a spectrum of disease ranging from the milder presentation observed in case 2 of our series (unilateral adrenal pathology only) to severe complications (bilateral adrenal haemorrhage, multisite disease and death) seen in the cases outlined by Blauenfeldt et al. and D'Agostino et al. $(9,10)$.

The most important clinical implication of adrenal haemorrhage or infarction is associated adrenal insufficiency, especially if there is bilateral adrenal involvement. Undiagnosed adrenal insufficiency can complicate the course of VITT, a potentially severe illness where an adequate stress response is required. An added complexity in the assessment and management of adrenal insufficiency in this setting is that patients may receive high doses of corticosteroids as part of the management of VITT (3). Twenty six per cent of patients in the recently published cohort study were treated with systemic glucocorticoids (i.v. methylprednisolone, oral or i.v. dexamethasone and oral prednisolone) (4). Cortisol levels in the acute setting may therefore not be easily interpretable. As demonstrated in case 1 , clinicians need to also recognise the potential for evolving adrenal involvement. An ongoing assessment for the development or progression of adrenal complications is especially important to consider when high dose 
corticosteroids used for treatment of VITT are stopped, as replacement doses of corticosteroids for adrenal insufficiency may still be required.

Although VITT post Oxford-AstraZeneca vaccination is rare (13), our experience highlights that clinicians need to be aware of potential adrenal complications (thrombosis/ infarction/haemorrhage). We suggest a safe and practical approach is to have a low threshold for starting empirical replacement with corticosteroids until reliable assessment of adrenal function can be performed, especially given the potential for evolving adrenal involvement.

\section{Declaration of interest}

We declare that there is no conflict of interest that could be perceived as prejudicing the impartiality of this case report.

\section{Funding}

This study did not receive any specific grant from any funding agency in the public, commercial or not-for-profit sector.

\section{Patient consent}

Written informed consent has been obtained from both patients for publication of the case report and accompanying images.

\section{Author contribution statement}

The authors are doctors involved in the care of case 1 (U S, A G, M S, B C) and case 2 ( $T$ C, E A, M S, B C). L D reviewed all images. All authors contributed to manuscript preparation.

\section{References}

1 Voysey M, Clemens SAC, Madhi SA, Weckx LY, Folegatti PM, Aley PK, Angus B, Baillie VL, Barnabas SL, Bhorat QE, et al. Safety and efficacy of the ChAdOx1 nCoV-19 vaccine (AZD1222) against SARS-CoV-2: an interim analysis of four randomised controlled trials in Brazil, South Africa, and the UK. Lancet 2021397 99-111. (https://doi.org/10.1016/ S0140-6736(20)32661-1)

2 Greinacher A, Thiele T, Warkentin TE, Weisser K, Kyrle PA \& Eichinger S. Thrombotic thrombocytopenia after ChAdOx1 nCov-19 vaccination. New England Journal of Medicine $20213842092-2101$. (https://doi.org/10.1056/NEJMoa2104840)
3 Pavord S, Lester W, Makris M, Scully M \& Hunt B. Guidance from the Expert Haematology Panel (EHP) on Covid-19 Vaccine-Induced Immune Thrombocytopenia and Thrombosis (VITT). UK: British Society for Haematology, 2021 [cited 2021 June 10]. (available at: https://b-s-h. org.uk/media/19718/guidance-v20-20210528-002.pdf)

4 Pavord S, Scully M, Hunt BJ, Lester W, Bagot C, Craven B, Rampotas A, Ambler G \& Makris M. Clinical features of vaccine-induced immune thrombocytopenia and thrombosis. New England Journal of Medicine 2021385 1680-1689. (https://doi.org/10.1056/NEJMoa2109908)

5 Douxfils J, Favresse J, Dogné JM, Lecompte T, Susen S, Cordonnier C, Lebreton A, Gosselin R, Sié P, Pernod G, et al. Hypotheses behind the very rare cases of thrombosis with thrombocytopenia syndrome after SARS-CoV-2 vaccination. Thrombosis Research 2021203 163-171. (https://doi.org/10.1016/j.thromres.2021.05.010)

6 Ketha S, Smithedajkul P, Vella A, Pruthi R, Wysokinski W \& McBane R. Adrenal haemorrhage due to heparin-induced thrombocytopenia. Thrombosis and Haemostasis 2013109 669-675. (https://doi. org/10.1160/TH12-11-0865)

7 Dobbie JW \& Symington T. The human adrenal gland with special reference to the vasculature. Journal of Endocrinology 196634 479-489. (https://doi.org/10.1677/joe.0.0340479)

8 United Kingdom Government. COVID-19 AstraZeneca Vaccine Analysis Print. UK: United Kingdom Government, 2021 [cited 2021 Aug 15]. (available at: https://assets.publishing.service.gov.uk/government/ uploads/system/uploads/attachment_data/file/1010799/COVID19_AstraZeneca_Vaccine_Analysis_Print_DLP_04.08.2021.pdf)

9 Blauenfeldt RA, Kristensen SR, Ernstsen SL, Kristensen CCH, Simonsen CZ \& Hvas AM. Thrombocytopenia with acute ischemic stroke and bleeding in a patient newly vaccinated with an adenoviral vector-based COVID-19 vaccine. Journal of Thrombosis and Haemostasis 202119 1771-1775. (https://doi.org/10.1111/jth.15347)

10 D’Agostino V, Caranci F, Negro A, Piscitelli V, Tuccillo B, Fasano F, Sirabella G, Marano I, Granata V, Grassi R, et al. A rare case of cerebral venous thrombosis and disseminated intravascular coagulation temporally associated to the COVID-19 vaccine administration Journal of Personalized Medicine 202111 285. (https://doi.org/10.3390/ jpm11040285)

11 Taylor P, Allen L, Shrikrishnapalasuriyar N, Stechman M \& Rees A. Vaccine-induced thrombosis and thrombocytopenia with bilateral adrenal haemorrhage. Clinical Endocrinology 2021 1-2. (https://doi. org/10.1111/cen.14548)

12 Varona JF, García-Isidro M, Moeinvaziri M, Ramos-López M \& Fernández-Domínguez M. Primary adrenal insufficiency associated with Oxford-AstraZeneca ChAdOx1 nCoV-19 vaccine-induced immune thrombotic thrombocytopenia (VITT). European Journal of Internal Medicine 202191 90-92. (https://doi.org/10.1016/j. ejim.2021.06.025)

13 United Kingdom Government. Coronavirus Vaccine - Weekly Summary of Yellow Card Reporting 2021. UK: United Kingdom Government, 2021 [cited 2021 June 10]. (available at: https://www.gov.uk/government/ publications/coronavirus-covid-19-vaccine-adverse-reactions/ coronavirus-vaccine-summary-of-yellow-card-reporting\#yellow-cardreports)

Received in final form 29 November 2021

Accepted 19 January 2022 